\title{
Long non-coding RNA H19 promotes the proliferation of fibroblasts in keloid scarring
}

\author{
JIE ZHANG ${ }^{1 *}$, CAI YUE LIU ${ }^{2 *}$, YUN WAN ${ }^{3}$, LI PENG $^{3}$, WEN FANG LI $^{4}$ and JIA XUAN QIU ${ }^{1}$

\begin{abstract}
${ }^{1}$ Department of Oral and Maxillofacial Surgery, The First Affiliated Hospital of Nanchang University, Nanchang, Jiangxi 330006; ${ }^{2}$ Department of Plastic Surgery, Changzheng Hospital, Second Military Medical University, Shanghai 200003; ${ }^{3}$ Department of Plastic Surgery, The First Affiliated Hospital of Nanchang University, Nanchang, Jiangxi 330006; ${ }^{4}$ Department of Plastic Surgery, Wenzhou Heping Plastic Surgery Hospital, Wenzhou, Zhejiang 325000, P.R. China
\end{abstract}

Received December 1, 2014; Accepted December 15, 2015

DOI: $10.3892 / \mathrm{ol} .2016 .4931$

\begin{abstract}
The expression of the long non-coding RNA (lncRNA) H19 is associated with proliferation in tumors. In order to investigate whether H19 may additionally mediate the proliferation of fibroblasts in human keloid disease, the present study collected samples from 24 subjects, including 8 with keloids, 8 with normal scars and 8 normal skin controls. Reverse transcription-polymerase chain reaction revealed that $\mathrm{H} 19$ levels were markedly increased in human keloids compared with normal scars and normal skin controls $(\mathrm{P}=0.017)$. In order to identify a potential role for $\mathrm{H} 19$ in the proliferative activity of human keloid fibroblasts, small interfering (si)RNA-mediated silencing experiments were performed. H19 siRNA treatment markedly inhibited the proliferation of keloid fibroblasts, as assessed by 3-(4,5-dimethylthiazol-2-yl)-2,5-diphenyltetrazolium bromide assay $(\mathrm{P}=0.008)$. In order to identify the signaling mediators that are regulated by H19 in keloid fibroblasts, the expression levels of mammalian target of rapamycin (mTOR) and vascular endothelial growth factor (VEGF) were examined using western blotting. The results confirmed that knockdown of H19 inhibited mTOR and VEGF expression. In summary, the results indicate that $\mathrm{H} 19$ may be associated with increased proliferative activity of keloid fibroblasts, which may be mediated by mTOR and VEGF.
\end{abstract}

Correspondence to: Dr Jia Xuan Qiu, Department of Oral and Maxillofacial Surgery, The First Affiliated Hospital of Nanchang University, 17 Yongwai Main Street, Nanchang, Jiangxi 330006, P.R. China

E-mail: qiujiaxuan@163.com

Dr Wen Fang Li, Department of Plastic Surgery, Wenzhou Heping Plastic Surgery Hospital, 312 East Ring Road, Wenzhou, Zhejiang 325000, P.R. China

E-mail:wenfanglee@126.com

*Contributed equally

Key words: keloid, long non-coding RNA H19, proliferation, mammalian target of rapamycin, vascular endothelial growth factor

\section{Introduction}

Keloid disease (KD), alternatively known as keloid scarring, is a type of fibrous tissue proliferation that commonly occurs following skin trauma or plastic surgery (1). Previous studies (2) have demonstrated that the pathogenesis of KD is associated with the functional activity of multiple signaling transduction pathways, genes and cytokines. Fibroblasts are the primary effector cells of KD tissue, the typical pathological features of which include excessive proliferation, disordered apoptosis and secretion of large amounts of extracellular matrix (3).

Long non-coding RNAs (lncRNAs) are a group of RNA molecules, which range in length from 200 to 100,000 nucleotides and do not encode any proteins (4). Numerous studies (5) have demonstrated that IncRNAs participate in various important regulatory processes, including transcriptional activation, transcriptional interference and intranuclear transport. Aberrant expression of the lncRNA H19 has been observed in various tumor types, suggesting potential oncogenic activity (6). Conversely, deletion of $\mathrm{H} 19$ reduces the proliferation and invasiveness of tumor cells (7). Given that H19 has a unique role in gene expression and tumorigenesis, the present study aimed to investigate the hypothesis that H19 may also be associated with KD. The expression of H19 RNA in KD tissue from patients was initially measured using reverse transcription-polymerase chain reaction (RT-PCR), and the association between $\mathrm{H} 19$ and proliferation of KD fibroblasts was subsequently investigated using small interfering (si)RNA transfection and pathway analysis. The results define the function of H19 in KD and suggest a signaling mechanism that may be associated with the proliferation of keloid fibroblasts.

\section{Materials and methods}

Study participants and sample collection. Tissue specimens were collected from $8 \mathrm{KD}$ patients who received treatment at The First Affiliated Hospital of Nanchang University (Nanchang, China) between May 2006 and June 2013. None of the patients had received any medical treatment prior to surgery. Informed consent was obtained from all patients prior to surgery, and the research protocol was approved by the Ethics Committee of The First Affiliated Hospital of Nanchang 
University (approval no., 2014D0307) prior to sample collection. The diagnosis of KD was confirmed by postoperative pathology, according to scar management practical guidelines $(8,9)$. As a control, an additional 8 cases of normal skin and 8 cases of normal mature scars were collected from the chest and back, which was similar to those of the KD cases.

Isolation and culture of fibroblasts. Freshly resected specimens were washed with normal saline three times. Following removal of the epithelial tissue, the specimens were cut into $2 \times 2-\mathrm{mm}$ tissue blocks and spread evenly across sterile flasks. Following the addition of $0.5 \mathrm{ml}$ Dulbecco's modified Eagle's medium (DMEM; InvivoGen, San Diego, CA, USA), the tissues were cultured at $37^{\circ} \mathrm{C}$ in an atmosphere of $5 \% \mathrm{CO}_{2}$ in an incubator for $4 \mathrm{~h}$. Subsequently, an additional $5 \mathrm{ml}$ DMEM and a small amount of fetal bovine serum (FBS; InvivoGen) were added, the flasks were rotated and culture was continued. The medium was replaced every 3-4 days. When the cultured fibroblasts grew to confluence, they were washed with phosphate-buffered saline, digested with $0.25 \%$ trypsin (InvivoGen) and passaged in fresh medium. Passage-three cells were used for subsequent experiments.

Liposome-mediated siRNA interference. In vitro-cultured fibroblasts were left untransfected (blank control group), or were transfected with H19 siRNA (H19 siRNA group) or a non-homologous siRNA (negative control group). The H19 siRNA was composed of a mixture of three siRNAs: H19-siRNA1, 5'-CCA ACAUCAAAGACACCAUdTdT-3'; H19-siRNA2, 5'-GCAGGA CAUGACAUGGUCCdTdT-3'; and H19-siRNA3, 5'-UAAGUC AUUUGCACUGGUUdTdT-3' (10). When cells grew to 60-80\% confluence, transfection was performed with Attractene Transfection Reagent (Qiagen, Inc., Valencia, CA, USA) according to the manufacturer's protocol ( 8 wells/group). Following $5 \mathrm{~h}$ of transfection, DMEM containing 20\% FBS was added, and cells were cultured at $37^{\circ} \mathrm{C}$ for $24 \mathrm{~h}$. Subsequently, the medium was replaced with fresh DMEM containing 10\% FBS, and cell culture was continued for 24-48 h prior to harvesting.

Detection of IncRNA H19 expression by RT-PCR. Total RNA was isolated from fresh tissue specimens $(100 \mathrm{mg})$ from each patient, or from cultured transfected cells harvested 1 day subsequent to fibroblast transfection. RNA was extracted using TRIzol $^{\circledR}$ reagent (Invitrogen; Thermo Fisher Scientific, Inc., Waltham, MA, USA) according to the manufacturer's protocol. Reverse transcription was performed with the SuperScript ${ }^{\circledR}$ II Reverse Transcriptase kit (Invitrogen; Thermo Fisher Scientific, Inc.) according to the manufacturer's protocol. Subsequently, PCR was performed in order to assess the expression of IncRNA H19 using the GeneAmp ${ }^{\circledR}$ PCR System 9700 (Applied Biosystems; Thermo Fisher Scientific, Inc.) using PrimeSTAR HS DNA Polymerase with GC Buffer (Takara Bio, Dalian, China). Glyceraldehyde-3 phosphate dehydrogenase (GAPDH) was used as the internal loading control. The primers were designed using Primer Premier 5.0 software (Premier Biosoft International, Palo Alto, CA, USA) and synthesized by Sangon Biotech Co. Ltd. (Shanghai, China) as follows: H19, forward 5'-TAC AACCACTGCACTACCTG-3' and reverse 5'-TGGAATGCT TGAAGGCTGCT-3'; and GAPDH, forward 5'-GGGAGCCAA AAGGGTCAT-3' and reverse 5'-GAGTCCTTCCACGATACC
AA-3'. The reaction conditions were $95^{\circ} \mathrm{C}$ for $10 \mathrm{~min}$, followed by 40 cycles of $95^{\circ} \mathrm{C}$ for $30 \mathrm{sec}$ and $60^{\circ} \mathrm{C}$ for $1 \mathrm{~min}$. The specificity of amplification products was confirmed using a negative control (no cDNA) and a RT-minus control (no RT). The relative expression of $\mathrm{H} 19$ in each group was quantified by agarose gel electrophoresis with ethidium bromide [Tiangen Biotech (Beijing) Co., Ltd., Beijing, China], followed by measurement of the optical density (OD) of the electrophoretic bands (NanoDrop 1000 Spectrophotometer; Thermo Fisher Scientific Inc., Wilmington, DE, USA). The DNA ladder was also obtained from Tiangen Biotech (Beijing) Co., Ltd.. Each specimen was tested 8 times.

Assessment of proliferation of KD fibroblasts by 3-(4,5dimethylthiazol-2-yl)-2,5-diphenyltetrazolium bromide (MTT) assay. The transfected fibroblasts were digested with trypsin and seeded as cell suspensions at a concentration of $1 \times 10^{4}$ cells/ml into 96 -well plates, with $100 \mu 1$ suspension per well. A total of $20 \mu 1$ MTT $(5 \mathrm{mg} / \mathrm{ml})$ was added to each of 8 wells per group, and culture was continued for 12, 24 or $48 \mathrm{~h}$. Following the end of this incubation period, the supernatants were discarded and $150 \mu 1$ dimethyl sulfoxide was added to each well. Plates were vibrated to dissolve the crystals thoroughly, and OD was measured at $490 \mathrm{~nm}$ (NanoDrop 1000 Spectrophotometer).

Detection of vascular endothelial growth factor (VEGF) and mammalian target of rapamycin (mTOR) expression in transfected fibroblasts by western blotting. Cells were harvested $48 \mathrm{~h}$ subsequent to transfection, and lysates were prepared using RIPA cell lysis reagent (Beyotime Institute of Biotechnology, Beijing, China) according to the manufacturer's protocol. Protein concentrations in lysates were quantified by the Bradford method (11), and equal amounts of protein were loaded onto sodium dodecyl sulfate-polyacrylamide gels. Following electrophoresis, the proteins were transferred to membranes using a wet transfer method, blocked with $1 \%$ bovine serum albumin in Tris-buffered saline containing $0.1 \%$ Tween 20 (TBST; Sangon Biotech Co. Ltd.) and incubated with primary antibodies (dilution, 1:500) overnight. The polyclonal goat anti-rabbit anti-VEGF (catalog no., sc-507), polyclonal goat anti-rabbit anti-mTOR (catalog no., sc-8319) and polyclonal goat anti-rabbit anti-caspase-3 (catalog no., sc-7148) antibodies were obtained from Santa Cruz Biotechnology, Inc. (Dallas, TX, USA). The internal reference, a polyclonal goat anti-rabbit anti-GAPDH antibody (dilution, 1:5,000; catalog no., G9545) was from Sigma-Aldrich (St. Louis, MO, USA). The membranes were washed in TBST and incubated with goat anti-rabbit horseradish peroxidase-labeled secondary antibody (dilution, 1:5,000; catalog no., CW0103S; ComWin Biotech Co., Ltd., Beijing, China) at room temperature for $1 \mathrm{~h}$. Protein bands were detected using Immoblion Western Chemiluminescent HRP Substrate (EMD Millipore, Billerica, MA,USA), and the intensity of the bands was analyzed using ImageJ software (National Institute of Health, Bethesda, MD, USA).

Statistical analysis. Data were analyzed by SPSS version 19.0 (IBM SPSS, Armonk, NY, USA) and are expressed as the mean \pm standard deviation. Comparisons were performed 
A

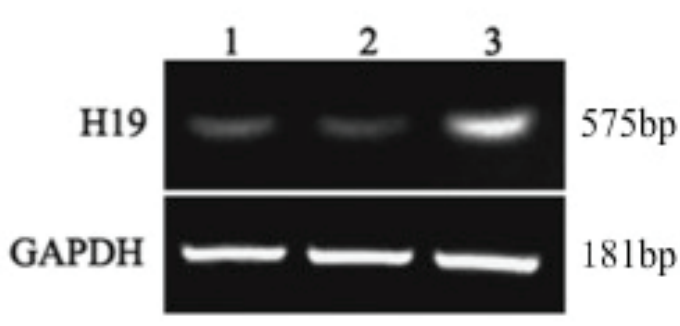

B

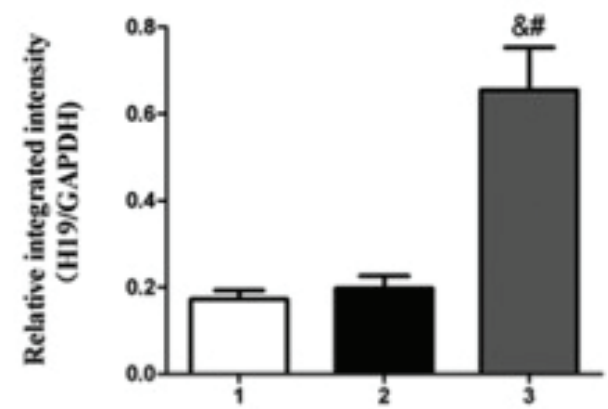

Figure 1. H19 expression is elevated in KD. H19 lncRNA expression in normal tissue (lane 1) and normal fibrous tissues (lane 2) was compared with that in KD tissue (lane 3) using RT-PCR. (A) Representative agarose gel showing RT-PCR results of one sample from each group is provided. GAPDH was assessed as an internal control. (B) The expression values (optical densities) of $\mathrm{H} 19$ relative to GAPDH expression were calculated for 8 samples from each group and expressed as the means \pm standard deviations. ${ }^{\&} \mathrm{P}<0.05$ compared with normal tissue; ${ }^{*} \mathrm{P}<0.05$ compared with mature fibrous tissue. KD, keloid disease; lncRNA, long non-coding RNA; RT-PCR, reverse transcription-polymerase chain reaction; GAPDH, glyceraldehyde-3-phosphate dehydrogenase.

using analysis of variance and the Student's $t$-test. Paired comparisons were performed by Fisher's least significant difference test. $\mathrm{P}<0.05$ was considered to indicate a statistically significant difference.

\section{Results}

Expression of IncRNA H19 is elevated in KD tissues. To determine whether $\mathrm{H} 19$ expression is associated with KD, tissue samples were collected from 24 study participants, including 8 with keloids, 8 with normal scars and 8 normal skin controls. The expression of lncRNA H19 was significantly increased in KD tissues $(0.6550 \pm 0.2767)$ compared with normal $(0.1725 \pm 0.0568)$ and mature $(0.1975 \pm 0.0808)$ fibrous tissues ( $\mathrm{P}=0.017$; Fig. 1). The results indicated that elevated H19 may be associated with KD. As keloid fibroblasts proliferate more rapidly compared with normal fibroblasts (12), it is possible that H19 may regulate proliferation in KD tissues.

$H 19$ regulates the proliferation of KD fibroblasts. In order to directly assess whether $\mathrm{H} 19$ is involved in the proliferation of keloid fibroblasts, RNA interference technology was used to silence the expression of $\mathrm{H} 19$ in cultured keloid fibroblasts. Reduced H19 RNA expression was confirmed at $24 \mathrm{~h}$ following transfection of specific H19 siRNA (si-H19; 0.0350 \pm 0.0 .0293 ) as compared with the blank $(0.6280 \pm 0.1880)$ or negative control siRNA (si-scramble; $0.5825 \pm 0.2440)$ groups $(\mathrm{P}=0.017$; Fig. 2A and B).

Furthermore, compared with si-scramble, fibroblast proliferation was significantly inhibited by si-H19 at 12, 24 and $48 \mathrm{~h}$ following transfection (Fig. 2C): Si-scramble vs. si-H19; $12 \mathrm{~h}$, $0.1982 \pm 0.0279$ vs. $0.1421 \pm 0.0153(\mathrm{P}<0.01) ; 24 \mathrm{~h}, 0.2563 \pm 0.05127$ vs. $0.2015 \pm 0.0281(\mathrm{P}<0.01)$; and $48 \mathrm{~h}, 0.3143 \pm 0.0984$ vs. $0.2312 \pm 0.0315(\mathrm{P}<0.05)$. These findings demonstrated that H19 may mediate proliferation in keloid fibroblasts.

$H 19$ mediates the expression of VEGF and mTOR. In order to identify proliferative signaling pathways that may be associated with H19 expression in keloid fibroblasts, western blotting was performed on two signaling molecules associated with keloid cell proliferation, VEGF and mTOR. Compared with the blank (VEGF, 0.950 \pm 0.1007 ; mTOR, 0.610 \pm 0.0972 ) and negative
(VEGF, 0.934 \pm 0.1119 ; mTOR, 0.634 \pm 0.0939 ) control groups, the expression levels of VEGF and mTOR were significantly decreased in H19 siRNA-transfected cells $(0.356 \pm 0.0808$, $\mathrm{P}=0.013$ and $0.184 \pm 0.06914, \mathrm{P}=0.009$, respectively; Fig. 3 ). These findings suggest a signaling pathway that may explain the effects of $\mathrm{H} 19$ on proliferation in KD.

\section{Discussion}

To the best of our knowledge, the present study is the first to demonstrate that the expression of the lncRNA H19 is abnormally increased in KD tissues. Additionally, using RNA-mediated silencing, it was directly demonstrated that H19 expression regulates the proliferation of keloid fibroblasts. Furthermore, the functions of several proteins that may serve as downstream regulators of $\mathrm{H} 19$ were investigated. The results demonstrate that knockdown of $\mathrm{H} 19$ is able to reduce the expression of mTOR and VEGF, which may explain the functional effects of H19 on keloid cell proliferation.

Reduced mTOR levels following H19 knockdown are consistent with its effects on proliferation. The mTOR/P70S6k signaling pathway is known to participate significantly in the pathogenesis of KD, and inhibition of mTOR activity may inhibit the proliferation of KD fibroblasts (13). mTOR is the target protein of rapamycin in mammals. Inhibition of mTOR activity may block the phosphorylation of P70S6K and eukaryotic translation initiation factor $4 \mathrm{E}$ (EIF4E)-binding protein 1, and prevent the release and transcription of EIF4E, thus inhibiting cell growth and proliferation (13). P70S6K is the ribosome 40s small subunit of the protein kinase S6K. Phosphorylation of S6K leads to translational initiation of 5'-terminal oligopyrimidine mRNA, the product of which controls the majority of the components of translation; these translational components have a significant role in cell growth and proliferation induced by mitogen stimulation (14). Therefore, the observation in the present study that downregulation of H19 RNA may markedly reduce the amount of mTOR expression suggested that H19 is able to control the proliferation of KD fibroblasts via the mTOR signaling pathway.

The effects of H19 knockdown on VEGF may additionally contribute to the control of proliferation. VEGF overexpression is a significant factor in the formation and progression 
A

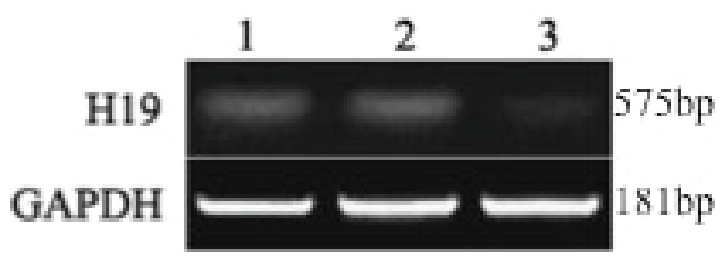

$\mathrm{C}$
B

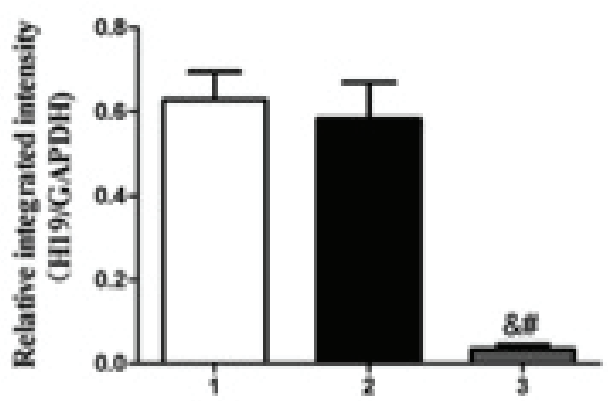

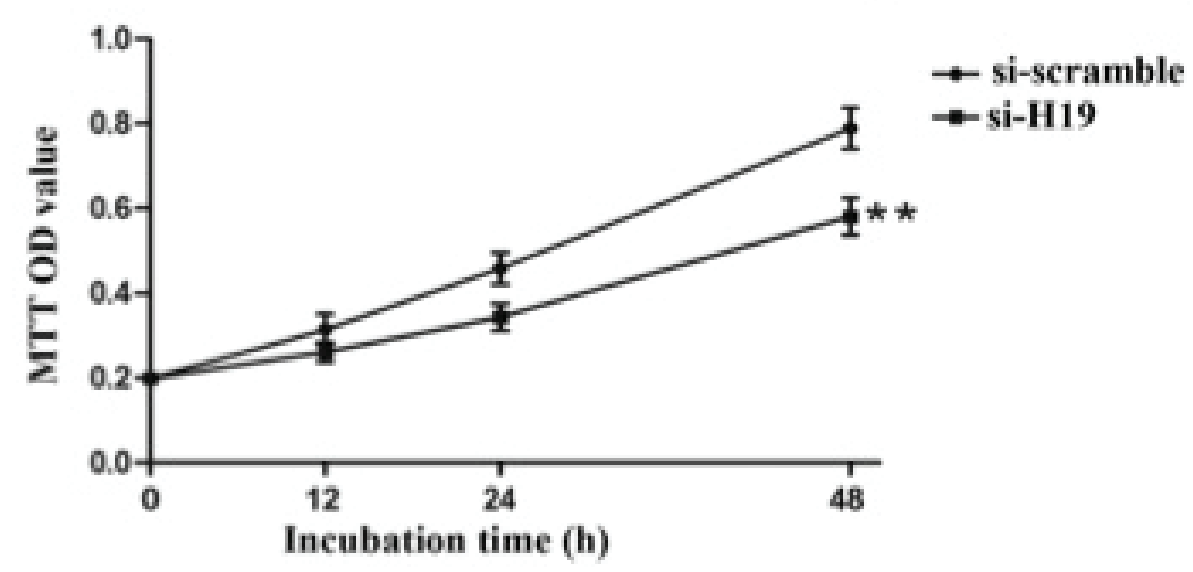

Figure 2. Knockdown of H19 expression suppresses the proliferation of keloid fibroblasts. (A and B) Keloid fibroblasts were transfected with blank control (lane 1), negative control siRNA (si-scramble; lane 2) or H19 siRNA (si-H19; lane 3), and the levels of H19 RNA expression relative to GAPDH mRNA expression were assessed by reverse transcription-polymerase chain reaction, gel electrophoresis and densitometry. (A) A representative gel is shown, in addition to (B) the semi-quantified results, presented as the mean \pm standard deviation of 8 experimental replicates. ${ }^{\&} \mathrm{P}<0.05$ compared with the blank control group; ${ }^{\#} \mathrm{P}<0.05$ compared with the negative control siRNA group. (C) Proliferation of keloid fibroblasts was assessed by MTT assay 12,24 or $48 \mathrm{~h}$ subsequent to transfection. Results represent the mean \pm standard deviation of 8 experimental replicates. ${ }^{* *} \mathrm{P}<0.01$ compared with negative control (si-scramble). siRNA, small interfering RNA; GAPDH, glyceraldehyde-3-phosphate dehydrogenase; MTT, 3-(4,5-dimethylthiazol-2-yl)-2,5-diphenyltetrazolium bromide; OD, optical density.

A

\section{VEGF} GAPDH 1

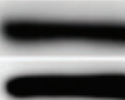

2

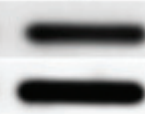

3

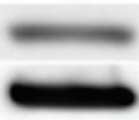

B

$43 \mathrm{kDa}$ $37 \mathrm{kDa}$

$$
\text { m-TOR }
$$

GAPDH
1

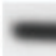

2
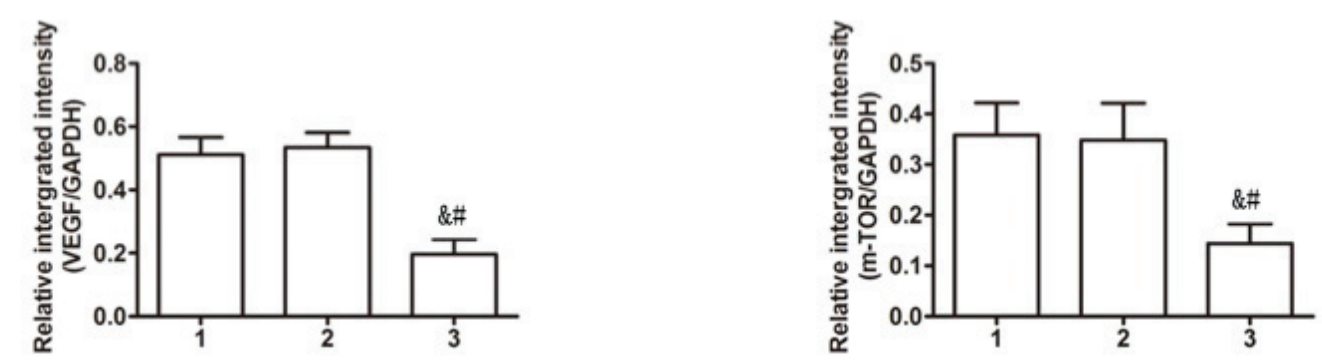

Figure 3. H19 knockdown reduces the expression of VEGF and mTOR in keloid fibroblasts. Expression of VEGF and mTOR was assessed by western blotting $24 \mathrm{~h}$ following transfection with blank control (lane 1), negative control siRNA (lane 2) or H19 siRNA (lane 3). (A and B) Representative results are shown, as well as quantification (mean \pm standard deviation) of the band densities relative to GAPDH obtained in 8 independent experiments. Results were standardized to an average of 1.0 in control cells. ${ }^{\circledR} \mathrm{P}<0.05$ compared with the blank control group; ${ }^{\sharp} \mathrm{P}<0.05$ compared with the negative control siRNA group. VEGF, vascular endothelial growth factor; mTOR, mammalian target of rapamycin; siRNA, small interfering RNA; GAPDH, glyceraldehyde-3-phosphate dehydrogenase.

of $\mathrm{KD}(15,16)$ and, consequently, inhibiting the production of VEGF in KD may reduce the number of newly formed vessels to inhibit KD growth (16). VEGF is a vascular endothelial cell-stimulating factor important in the formation of new vessels (17). It is expressed widely in the normal human body (18). Under normal physiological conditions, the expression of VEGF is relatively low; however, its expression is increased under certain pathological conditions, including in tumor tissues (19-21). In addition to promoting vascular formation, VEGF increases the blood supply and repair of damaged tissues and vascular endothelial cells (17). Previous studies have demonstrated that full-length VEGF is expressed in KD 
tissues, primarily in keratinocytes and fibroblasts $(22,23)$. In the present study, it was demonstrated that a reduction in $\mathrm{H} 19$ expression inhibited the formation of VEGF, thus inhibiting the proliferation of KD fibroblasts. These findings indicate that $\mathrm{H} 19$ is a significant factor regulating the generation of VEGF from fibroblasts.

Previous studies have revealed that the occurrence of pathological KD may be associated with abnormalities in additional signaling transduction pathways, including c-Myc and E2F transcription factor 1 (E2F1) $(24,25)$. The expression of the proto-oncogene c-Myc is markedly elevated in KD tissue (25). c-Myc is thought to be closely associated with cell proliferation and differentiation, primarily via a mechanism involving the activation of growth factors, receptors and intracellular signaling molecules (26). A previous study reported that c-Myc was able to induce the expression of $\mathrm{H} 19$ lncRNA (27). Therefore, H19 expression may be regulated by $\mathrm{c}-\mathrm{Myc}$ in keloid fibroblasts. The expression of E2F1 protein in $\mathrm{KD}$ tissues is also elevated compared with normal skin tissue, indicating that E2F1 has a significant role in fibroblast differentiation, proliferation or phenotype transformation and collagen synthesis (28). In addition, E2F1 is capable of inducing the expression of H19 (29). Thus, we hypothesize that H19 expression in KD fibroblasts may be regulated via c-Myc and E2F1, and the activation of H19 may have a significant role in the proliferation of fibroblasts in $\mathrm{KD}$, via downstream effects on mTOR and VEGF.

In conclusion, the current study demonstrated that H19 is overexpressed in $\mathrm{KD}$, and that this overexpression is closely associated with the proliferation of fibroblasts in KD. Downregulation of $\mathrm{H} 19$ expression was able to inhibit the generation of mTOR and VEGF, thus inhibiting the proliferation of KD fibroblasts. These findings indicate that H19 may serve as a novel target for the treatment of KD.

\section{Acknowledgements}

The present study was supported by the National Natural Science Foundation of China (grant no. 81460295) and Jiangxi Provincial Health and Family Planning Commission Science and Technology Project (grant no. 20155201).

\section{References}

1. Alster TS and Tanzi EL: Hypertrophic scars and keloids: Etiology and management. Am J Clin Dermatol 4: 235-243, 2003.

2. Wolfram D, Tzankov A, Pülzl P and Piza-Katzer H: Hypertrophic scars and keloids - a review of their pathophysiology, risk factors, and therapeutic management. Dermatol Surg 35: 171-181, 2009.

3. Mofikoya BO, Adeyemo WL and Abdus-salam AA: Keloid and hypertrophic scars: A review of recent developments in pathogenesis and management. Nig Q J Hosp Med 17: 134-139, 2007.

4. Gibb EA, Brown CJ and Lam WL: The functional role of long non-coding RNA in human carcinomas. Mol Cancer 10: 38, 2011

5. Prensner JR and Chinnaiyan AM: The emergence of lncRNAs in cancer biology. Cancer Discov 1: 391-407, 2011.

6. Matouk IJ, DeGroot N, Mezan S, Ayesh S, Abu-lail R, Hochberg A and Galun E: The H19 non-coding RNA is essential for human tumor growth. PLoS One 2: e845, 2007.

7. Matouk I, Raveh E, Ohana P, Lail RA, Gershtain E, Gilon M, De Groot N, Czerniak A and Hochberg A: The increasing complexity of the oncofetal h19 gene locus: Functional dissection and therapeutic intervention. Int J Mol Sci 14: 4298-4316, 2013.
8. Lee JY, Yang CC, Chao SC and Wong TW: Histopathological differential diagnosis of keloid and hypertrophic scar. Am J Dermatopathol 26: 379-384, 2004.

9. Monstrey S, Middelkoop E, Vranckx JJ, Bassetto F, Ziegler UE, Meaume S and Téot L: Updated scar management practical guidelines: Non-invasive and invasive measures. J Plast Reconstr Aesthet Surg 67: 1017-1025, 2014.

10. Yang F, Bi J, Xue X, Zheng L, Zhi K, Hua J and Fang G: Upregulated long non-coding RNA H19 contributes to proliferation of gastric cancer cells. FEBS J 279: 3159-3165, 2012.

11. Kruger NJ: The Bradford method for protein quantitation. Methods Mol Biol 32: 9-15, 1994.

12. Calderon M, Lawrence WT and Banes AJ: Increased proliferation in keloid fibroblasts wounded in vitro. J Surg Res 61: 343-347, 1996.

13. Ong CT, Khoo YT, Mukhopadhyay A, Do DV, Lim IJ, Aalami O and Phan TT: mTOR as a potential therapeutic target for treatment of keloids and excessive scars. Exp Dermatol 16: 394-404, 2007.

14. Laplante M and Sabatini DM: mTOR signaling in growth control and disease. Cell 149: 274-293, 2012.

15. Clark JA, Leung KS, Cheng JC and Leung PC: The hypertrophic scar and microcirculation properties. Burns 22: 447-450, 1996.

16. Wu WS, Wang FS, Yang KD, Huang CC and Kuo YR: Dexamethasone induction of keloid regression through effective suppression of VEGF expression and keloid fibroblast proliferation. J Invest Dermatol 126: 1264-1271, 2006.

17. Ferrara N, Gerber HP and LeCouter J: The biology of VEGF and its receptors. Nat Med 9: 669-676, 2003.

18. Crafts TD, Jensen AR, Blocher-Smith EC and Markel TA: Vascular endothelial growth factor: Therapeutic possibilities and challenges for the treatment of ischemia. Cytokine 71: 385-393, 2015.

19. Otrock ZK, Makarem JA and Shamseddine AI: Vascular endothelial growth factor family of ligands and receptors: Review. Blood Cells Mol Dis 38: 258-268, 2007.

20. Otrock ZK, Mahfouz RA, Makarem JA and Shamseddine AI: Understanding the biology of angiogenesis: Review of the most important molecular mechanisms. Blood Cells Mol Dis 39: 212-220, 2007.

21. Farhat FS, Tfayli A, Fakhruddin N, Mahfouz R, Otrock ZK, Alameddine RS, Awada AH and Shamseddine A: Expression, prognostic and predictive impact of VEGF and bFGF in non-small cell lung cancer. Crit Rev Oncol Hematol 84: 149-160, 2012.

22. Trompezinski S, Denis A, Vinche A, Schmitt D and Viac J: IL-4 and interferon-gamma differentially modulate vascular endothelial growth factor release from normal human keratinocytes and fibroblasts. Exp Dermatol 11: 224-231, 2002.

23. Trompezinski S, Berthier-Vergnes O, Denis A, Schmitt D and Viac J: Comparative expression of vascular endothelial growth factor family members, VEGF-B, -C and -D, by normal human keratinocytes and fibroblasts. Exp Dermatol 13: 98-105, 2004.

24. De Felice B, Garbi C, Wilson RR, Santoriello M and Nacca M: Effect of selenocystine on gene expression profiles in human keloid fibroblasts. Genomics 97: 265-276, 2011.

25. Hu Z, Lou L and Luo S: Experimental study of the expression of c-myc, c-fos and proto-oncogenes on hypertrophic and scars. Zhonghua Zheng Xing Wai Ke Za Zhi 18: 165-167, 2002 (In Chinese).

26. Bretones G, Delgado MD and León J: Myc and cell cycle control. Biochim Biophys Acta 1849: 506-516, 2014.

27. Barsyte-Lovejoy D, Lau SK, Boutros PC, Khosravi F, Jurisica I, Andrulis IL, Tsao MS and Penn LZ: The c-Myc oncogene directly induces the H19 noncoding RNA by allele-specific binding to potentiate tumorigenesis. Cancer Res 66: 5330-5337, 2006.

28. Luo X, Pan Q, Liu L and Chegini N: Genomic and proteomic profiling II: Comparative assessment of gene expression profiles in leiomyomas, keloids, and surgically-induced scars. Reprod Biol Endocrinol 5: 35, 2007.

29. Berteaux N, Lottin S, Monté D, Pinte S, Quatannens B, Coll J, Hondermarck H, Curgy JJ, Dugimont T and Adriaenssens E: H19 mRNA-like noncoding RNA promotes breast cancer cell proliferation through positive control by E2F1. J Biol Chem 280: 29625-29636, 2005 . 